\title{
An Advanced LTE Architecture for Multimedia CDN
}

\author{
Maryam M. Nazari Z \\ Shiraz University of Technology \\ Shiraz, Iran
}

\author{
Mostafa Borhani \\ Shahid Beheshti University \\ Tehran, Iran, Corresponding author
}

\begin{abstract}
One of the most important requirements of multimedia providers in the delivery of content requested by users with assurance of Quality of Service (QoS) and Quality of User Experience (QoE) in Long-Term Evolution (LTE) networks. The main focus of this paper is to delineate the overall architecture of internal communications and unified Content Distribution Networks (CDNs) from the perspective and interfaces available in LTE network architecture. The LTE Evolved Packet System (EPS) is customized for multimedia delivery and the elements of content delivery network interaction are introduced by this manuscript. The proposed architecture for CDN over LTE networks is analyzed to ensure QoS. Based on the evaluation process, the architecture is suitable for the distribution of live and on-demand multimedia content for simultaneous distribution over LTE networks with QoS constraints.
\end{abstract}

Keywords-LTE Networks, Content Delivery Networks, QoS, QoE, Interactive Services, Multimedia

\section{INTRODUCTION}

New ways of transmitting multimedia content over the Internet have been growing. Today, many live events are more easily accessible to interested users than ever before. The growth of smartphones, pockets, and high bandwidth capabilities enables users to easily access their desired content anytime and anywhere through mobile technologies.

Multimedia Messaging Service (MMS), video calls with data, text and motion/ static graphics, and multimedia video delivery (broadcasting services and video-on-demand) are some of the main multimedia services in an LTE system. Different types of multimedia content such as static content (text, graphics, and data), continuous (dynamic) content (video, audio, voice) or combined content should be properly delivered to the end consumer by LTE subsystems. The file delivery and the streaming (continuous) transmission are the common delivery approaches for multimedia content [1].

Many powerful platforms, such as YouTube, use CDNs to deliver content to their users. The overall architecture for interconnecting and integrating CDNs from the standpoints and interfaces available in LTE network architecture to achieve QoS assurance is the most challenging issue to achieve. This requires identifying the equipment needed to distribute content across large networks whose architecture provides them with these requirements.

The LTE network architecture should meet the abovementioned requirements. The main actors in this segment are telecommunications companies that have subsidiaries for mobile communications for their traditional Internet Service Provider (ISP) business as well as for the provision of interactive television services (IPTV). Also, multinational telecommunications companies intend to deliver content to all their national subsidiaries, and a group of ISPs that intend to provide partnerships to their subscribers over QoS guaranteed network privacy.

Before proposing the architecture of content distribution networks, we need to have a look at some of the technical terms associated with these networks. Fig. 1 is a diagram of the concept of content distribution networks. In general, content distribution operations take place in three stages: acquisition, deployment, and delivery [2].

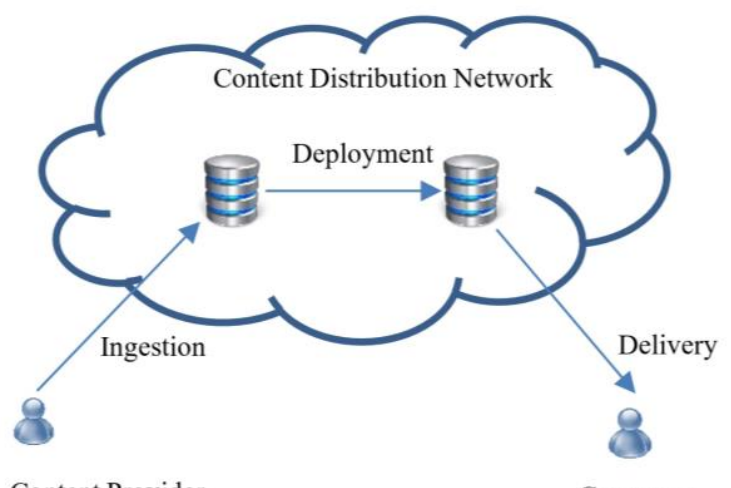

Content Provider Consumer

Fig. 1. Outline of the concept of content distribution and delivery

The process of putting new content on CDN by a content provider is called Ingestion. Once the content is uploaded and uploaded, the content is distributed to the CDN cache, which refers to the concept of content deployment. In the final step, the content is requested by the customer and the content is delivered to them.

The mobile centralized core networks are typically in the backbone network and usually are deployed in regional core COs (Central Office) in provincial capital cities. So, edge nodes of mobile CDN cannot be deployed in the vicinity of end-users [3].

Presenting the architecture of distributed CDNs to support QoS assurance, the LTE networks are reviewed by this section and then discuss the main challenges in implementing CDNs in the context of LTE networks. Nowadays, network capacity will be exceeded several times daily, resulting in loss of service for users [4]. The greater content and quality demand from the network should be provided for larger and higher resolution screens, greater processing power and reduced cost of smartphone and tablet devices [5] [6]. 
Fig. 2 shows a representation of the most important goals of the LTE network [7]. The main purpose of LTE networks is to provide a variety of data services over the packet switching platform and the most affordable Internet protocol.

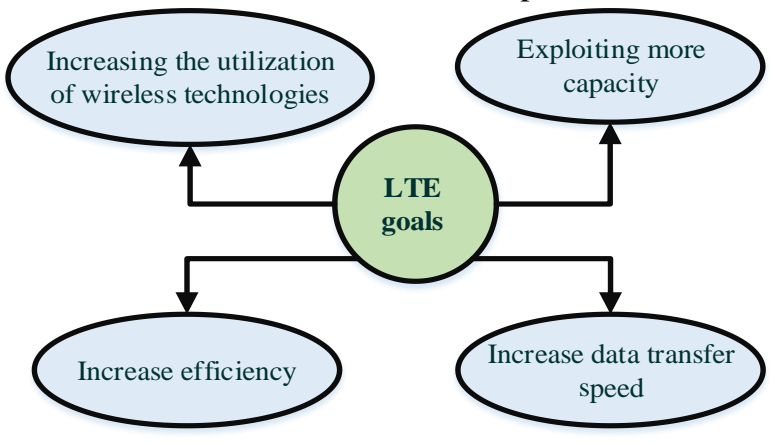

Fig. 2. The most important goals of the LTE network

In the network kernel segment, the network structure and nodes are quite different from the previous kernels, known as the Evolved Packet Core (EPC). As such, the whole architecture of the LTE network, in the access and kernel segment, is quite different from the current networks, collectively referred to as System Architecture Evolution (SAE). As such, the LTE architecture has created an Evolved Packet System (EPS).

In EPS, any traffic, including voice and data, is mounted on different carriers on the network and transmitted to the user through the Packet Data Network (PDN). Carrier is a packet of data that is generated according to the Internet protocol in different network interfaces and has a predefined QoS depending on the type of traffic it carries. The delivery carriers are done by different processes of the kernel and access sectors. The EPS network provides the user with access to the Internet and running services such as VoIP. Each EPS carrier is identified by its QoS. In the LTE network, it is possible to create multiple carriers for one user at a time to enable different QoS streams with different PDNs. All processes related to establishing and communicating are supported by different EPS network elements with different responsibilities.

The rest of the paper is organized as follows: the proposed architecture for multimedia content delivery in LTE networks based on the main components and their interactions is proposed in Section II in detail. Section III provides an analysis of the requirements of the proposed architecture based on internal domain QoS signaling, resource-layer synchronization, and SLA usage. Based on these studies, most of the functions required for CDNs are available on LTE networks by the proposed architecture which is presented in Conclusion Section

II. THE PROPOSED ARCHITECTURE OF CDNS ON LTE

One of the most important requirements of CDN networks is the QoS guarantee. CDNs need to support QoS metrics and reporting of QoS observations on the network. Also, it supports the implementation of the QoS rules and regulations and the sending of relevant information to target groups within the core tasks of CDNs.

These networks should be able to exchange QoS information based on a specific set of important parameters. The content distribution must be monitored to accurately measure the observed QoS. Content should also be linked to meta-specifications that describe QoS requirements. Compliance with this objective must necessarily be achieved if the relevant network rules and guidelines require a specific QoS.

There are different types of content on CDN networks, each with its own set of requirements for QoS guarantee, support for delivery methods, and additional infrastructure over the network [8] [9]. CDN should also support file downloads and other multimedia streams, regardless of video delivery.

Internal communications CDNs include communications between the internal equipment of a local CDN and communications with other local content distribution networks. In addition to hardware, we have a set of rules, configuration files, and public/private keys for internal CDN communications that guarantee the interaction of different local CDNs. Each local CDN contains the following components, which are implemented as hardware and software interactions that ensure proper interactions between them by sharing configuration files.

Remote Deployment: The main task of this section is to deliver content located within the local CDN to other CDN members connected to the network. Also, the content received from telecommunications elements in other CDNs is transferred to the local delivery component by the telecommunications component. This component should be able to support different modes of content use.

Resources Store: This section is responsible for downloading content files and related metadata. It also responds to requested information about the location and content metadata as a reference server.

Local Delivery: This section provides an initial customer service agent for content customers. Digital Receiver Service Providers are an example of equipment in this segment to provide IPTV services to subscribers. Web browsing media directories such as YouTube can also be maintained by service providers, allowing customers to easily access the content provided. Actual delivery mechanisms depend on the type of content being distributed. Also, some content on the CDN may require specific QoS requirements, which in turn is specified in the resource section.

Local QoS Authority: This unit defines QoS features as a Service Level Agreement (SLA) or Service Level Identifier (SLS) with relevant partners. Also, the user interfaces for querying QoS features for connecting with peers. This is done through local information received from local QoS authorities on other CDNs and stores measured QoS information. The most important QoS features to monitor include: output (capacity), delay, packet arrival times (Jitter), and packet loss. Returns the amount of data that can be transmitted from the content distribution server to the content client over a specified period. Higher quality content has more volume and content type depends on real-time playback, so more bandwidth needs to be allocated for transmission. Also, the statistical properties of the criteria must be recorded. An audit mechanism may be required depending on the type of contract between content providers and CDN operators. It should be noted that having a local QoS reference does not mean that the overall architecture requires QoS support. But if the ISP 
supports QoS, the architecture provides mechanisms to disseminate this information.

Logging Information: This section receives messages from other logged-in elements. It also provides the ability to search through recorded data and provides an interface to organize the reporting framework. Privacy rules in this section should apply.

Accounting: An audit module is a tool used to calculate service bills. An interface is defined for the local charging infrastructure that depends on the business model of the CDN provider.

To understand the interaction of CDN network elements in the architecture, the relationships between each of the architectural elements are numbered. In this section, descriptions of each are given with their respective numbers. Fig. 3 shows some of the internal communications of CDNs.

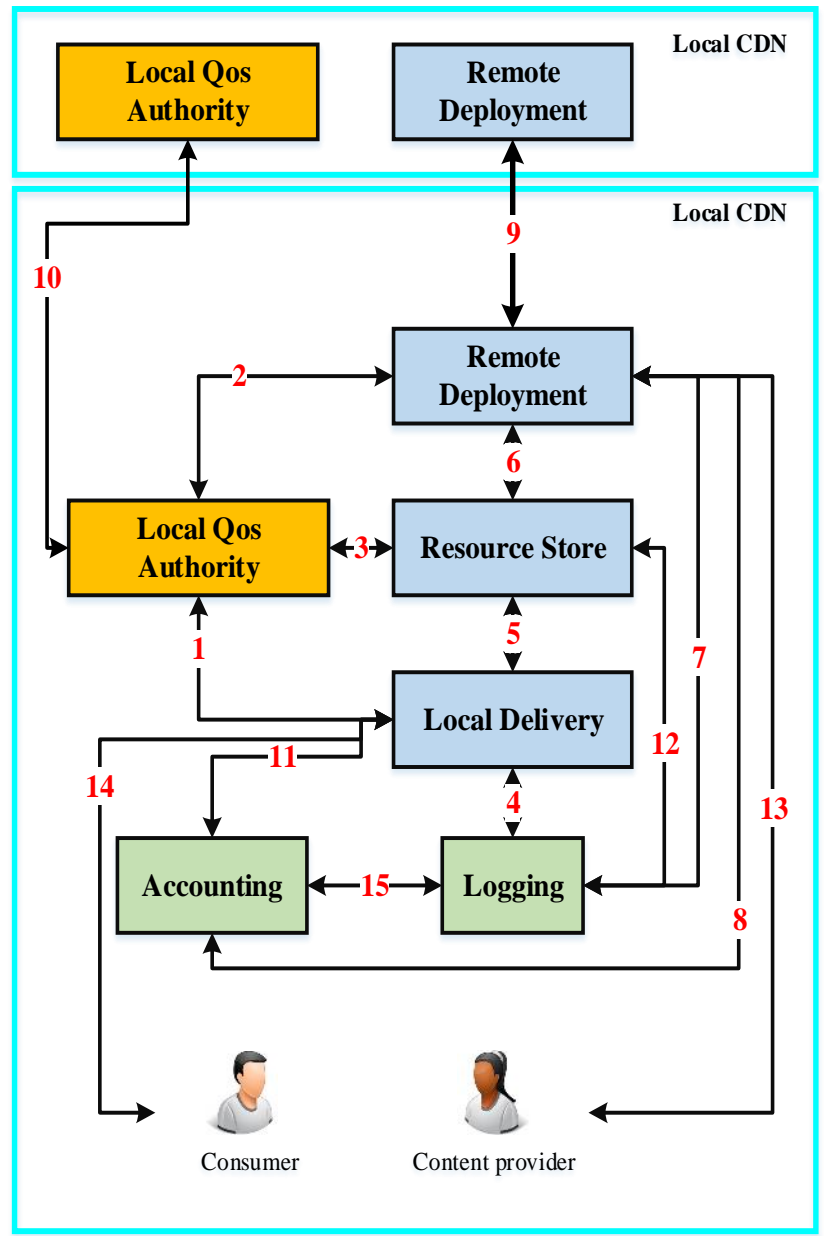

Fig. 3. Internal communications of content delivery and distribution networks

In this figure, the main components and their interactions for the proposed CDN architecture are presented.

(1) Local QoS Monitoring: During content delivery to the local CDN, the observed QoS criteria are monitored and delivered to the local QoS reference for storage and aggregation.

(2) QoS control: Before sending the content to the local CDN or a remote $\mathrm{CDN}$, the local QoS criterion is required to check whether the QoS requirements are met.
(3) Local QoS: If the metadata of the distributed content identifies QoS constraints, resource storage will monitor local QoS criteria at regular intervals to search for perceived QoS criteria for this content and record the results of this interaction Can be.

(4) Delivery Log: Content delivery information will be recorded on the local CDN.

(5) Content Request: The local delivery element for the content requests and delivers the location and metadata.

(6) Local Content Placement: Recent imported content and its metadata are stored in the resource cache. If the QoS content constraints are required, a scheduling census mechanism operates during the broadcast [9].

(7) Data Recording: If the content is hosted on a CDN by the content provider or deployed by a remote $\mathrm{CDN}$ on the local CDN, the information is recorded.

(8) Audit: When new content is received, the relevant message will be sent from the audit element if the delivery of this content requires accounting. Also, if the deployment of content to this CDN involves costs, it is processed by the auditing department. It should be noted that every audit operation is recorded.

(9) Remote Deployment: Content metadata may be from any of the local whitelists or blacklists of specific CDNs. Depending on the type of information and QoS, the content is deployed on remote CDNs. Content is stored in remote $\mathrm{CDN}$ resources. If the deployment of content in this CDN is included, the audit element sends a message. The content deployment process will also be recorded.

(10) QoS Information Dissemination: QoS features are distributed from local CDN to other CDNs.

(11) Local Audit: If the content is requested from the local delivery element and the content metadata is received from the source cache, the local deployment component audit operation confirms that the requesting user is authorized to receive the content. If the user is not allowed, the metadata of the content determines the instant purchase opportunity and the audit element takes care of the operations required.

(12) Resource Recording: Content placement and metadata updates are recorded.

(13) Content Placement: The content provider intends to distribute the content and upload its content and metadata related to remote startup components. The deployment component guarantees the required QoS constraints on all local CDNs. The audit element is informed of the content received. Also, content is stored in source storage and remotely deployed CDNs. Status records for all activities.

(14) Content Delivery: The customer requests the content from the local delivery element. Position the content and metadata from the source store request and local delivery element Perform user access and audit operations as needed. QoS is monitored when data transfers are initiated to the client and reports are sent to the local QoS authority. During these steps, data logging operations are also performed.

(15) Audit Record: All audit operations will be recorded. 


\section{ANALYSIS OF PROPOSED CONTENT DELIVERY ON LTE}

The proposed networking scenario assumed in Fig. 4, which involves the user connected to the packet-core evolved through LTE technology or other cellular technologies. The architecture of Fig. 4 consists of two main parts, the kernel, and the access network. The kernel consists of a large number of logical nodes. In the access section, however, there is only one node called the NodeB extension node known as eNodeB, to which the UEs connected to it. EPC requires the integration of different types of cellular access.

The main logic nodes in the EPC are PDN gateway (PGW), service gateway (S-GW), Mobility Management (MME), Evolved Cellular Positioning Service (E-SMLC). The two core logic entities are the eNodeB and the SAE-GW user side. eNodeB includes end-user radio interface and SAE-GW communication to an external network such as the Internet. This is a great simplification compared to a cellular network such as UMTS, in which there are parallel structures for circuit switching and data path switching. SAE-GW is a combination of the functions of two logical entities S-GW and PDN-GW or P-GW. All user IP packets are transmitted over $\mathrm{S}-\mathrm{GW}$. This node is the local controller for sending data carriers when moving users across the network and moving between eNodeBs.

Carrier information is stored in the UE idle state in S-GW and temporary user data is buffered downlink paths to initiate MME call for UE carriers. Collecting information for charging and disconnecting with legal licenses on the destination network is one of the tasks of S-GW.

In addition to the above, the user and its $\mathrm{HO}$ switch between the LTE network and networks using other 3GPP technologies, such as GPRS, and UMTS by S-GW.

Major tasks of PDN-GW include assigning IP addresses to the UE, enforcing QoS policies, facilitating HO between 3GPP networks (eg GSM or UMTS) and non-3GPP networks (eg WIFI or WINMAX). Other tasks of this unit include maintaining carrier quality, charging based on information received from PCRF, filtering downlink user IP packets based on their requested quality, and organizing QoS execution for guaranteed bit rate carriers.

From the signaling side is the Motion Management Unit or MME. This unit controls the exchange of information between the user terminals and the main network. It also makes the necessary adjustments and reallocation of carriers, which refers to the concept of user level in EPS. The most important tasks of an MME are carrier management, communication management, Non-Access Stratum (NAS) control.

Another control node is HSS, which stores user data for auditing and other purposes. This node holds all user information on the LTE network, including user specifications, user-requested quality profiles for a variety of services, and restrictions on access to roaming. HSS also stores data about the data network that the user can connect to in the form of an access point name (APN) or PDN address. Also, it stores dynamic information such as the MME identification code that the user is currently connected to or registered with for routing processes.

PCRF is one of the most important parts of this article. Apart from charging various user services, it provides information about each subscriber and provides actions such as running QoS on gateway nodes such as PDN-GW. The desired quality of each carrier is verified by PCRF and the matching of the requested quality and service of the user with their subscription profile at this node is checked.

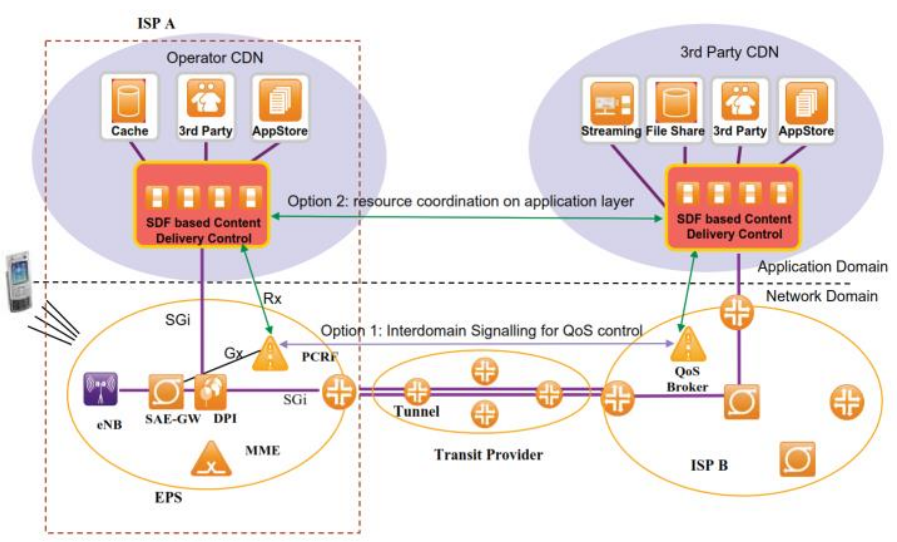

Fig. 4. The proposed architecture for multimedia content delivery over LTE network platform

This user intends to consume live multimedia streaming on their mobile device (EPS Mobile Broadband for ISP A). The stream is in some locations owned by a CDN that connects to the ISP B domain. Both ISP A and ISP B networks are connected via another ISP.

QoS should not be a problem for transmission networks, as there is generally enough bandwidth available and also the jitter provided by this network is low enough to deliver delaysensitive traffic with good user experience. Also, it should be assumed that the content provider creates at least SLAs with various ISPs, including ISP A and ISP B, which regulate more details of end-to-end delivery such as QoS support on the respective networks.

The PCRF in this network is responsible for controlling access to network resources in the EPC. Monitoring uses a traffic inspection element such as DPI, which is shared with the gateway node in the user position, usually PDN-GW. ERF in PDN-GW via the Gx interface provides feedback to PCRF about using current sources.

Part of the local delivery element that manages the flow of resources is related to PCEF in PDN-GW. Not all functions and components described for the CDN architecture have an equivalent EPC. Therefore, it is assumed that its implementation is part of the $\mathrm{CDN}$ coverage.

The available EPC functions are used for auditing and charging for network-related tasks. However, the proposed CDN audit module is used to charge certain content. Because ISPs and content providers usually have separate value chains, integrating these functions may not be possible. Based on the present EPC network architecture and the CDN architecture, there are three general ways to support QoS for live streaming along with long-distance multiple domains.

Interdomain Signaling for QoS: The solution based on this concept requires PCRF to process QoS information from external domains. Various protocols have been proposed for the exchange of such information, such as the BGP routing protocol or the management of modified internal domain 
markup routes for MPLS [11]. Assuming that IP transmission networks have sufficient capacity to transmit appropriate media streams to assure the quality of the experience, the signaling information must be exchanged between the EPS network and the previous-hop domain or source coverage stream (eg ISP B network). For this purpose, QoS information may be transmitted between ISP domains associated with different coverage nodes.

However, there are some notable concerns about this method. There is no standard protocol for QoS control at the internal domain level so far. Also, the implementation is unlikely to be due to the ISP's lack of demand for such a mechanism. The PCRF should also be expanded with internal bandwidth communication function and support QoS data transfer as far as possible. The changes required have a significant impact on the EPC architecture.

Application Layer Coordination: The best way to implement CDNs on LTE networks should be as limited as possible to the EPS architecture in the first instance. The bandwidth deployment function should be applied to the application layer, ie CDN coverage. The features set for QoS negotiation should be supported based on a common platform used for all CDN networks nationwide. QoS assurance in this context should be unique in the ISP domains associated with CDN coverage, and QoS differentiation needs to be based on startup information between the CDN control framework and the underground network infrastructure. Within the EPC, the existing Rx interface for the exchange of control information between the application function and the PCRF, along with some parameters developed for further analysis, can be used to support the concept. The parameters needed to coordinate resources in the application layer need further improvement by using artificial intelligence [12] and can be addressed in future research.

The main challenge for the approach presented is in the application layer, such as running a QoS service as part of a trusted shared CDN platform. At first glance, this approach is preferred because the changes required in the EPC are relatively small and can be used over a relatively short period. In some cases, the end-to-end path between the source and the synchronization of a stream does not extend beyond the domain of the two ISPs. No transfer providers are involved. QoS support can be facilitated through SLAs between the two domains. Therefore, there is no need to exchange dynamic control information. Larger ISPs, for example, may provide cellular access and access to fixed networks. Common network infrastructures, including node-connected transmission networks, are used for mobile networks and fixed-core networks. The connection is made through private exchange points. This approach does not require much change in EPS, ISP networks or application level. However, its application is limited due to the specific limitations of the scenario in question and the inability to respond to dynamic network conditions.

\section{CONCLUSION}

This paper proposed an advanced architecture for video delivery over LTE networks. The requirements for content delivery by content distribution networks over the LTE network context to achieve QoS for content distribution were clarified by this study. The proposed architecture leads CDNs to achieve QoS by voluntarily work together in LTE networks. We identified the functions needed to interact with video delivery networks with LTE networks. This manuscript also provides an outline of the LTE broadband architecture and analyze its needs for internal domain QoS signaling, resourcelayer synchronization, and SLA usage. This conceptual study concludes that fortunately most of the functions required for CDNs are available on LTE networks by the proposed architecture.

\section{REFERENCES}

[1] 3GPP TS 26.346 Multimedia Broadcast/Multicast Service (MBMS) Protocols and codecs, V16.3.0, 2019

[2] S. Retal, M. Bagaa, T. Taleb, and H. Flinck, "Content delivery network slicing: Qoe and cost awareness," in Communications (ICC), 2017 IEEE International Conference on. IEEE, 2017, pp. 1-6.

[3] 3GPP TR23.401. General Packet Radio Service (GPRS) enhancements for Evolved Universal Terrestrial Radio Access Network (E-UTRAN) access. V15.4.0, June 2018.

[4] IGR. Content for All-The Potential for LTE Broadcast/eMBMS White Paper. Accessed on Jan. 9, 2013. [Online]. Available: https://igrinc.com/Downloads/?ID=927

[5] James Miller, Roadmap for 5G in 3GPP, 2015. [Online]. Available: http://www.3gpp.org/ftp/workshop/2015-09 17_18_RAN_5G/Docs/RWS-150030.zip

[6] M Borhani, SM Atarodi, A new channel estimator design for nextgeneration high-speed mobile data communications based on OFDM The 12nd Iranian Conference on Electrical Engineering, 2004.

[7] Wiley, LTE The UMTS Long Term Evolution From Theory to Practice, 2nd.Edition, 2011.

[8] B. Tullemans, "Technical Practices for a Multi-CDN Distribution Strategy," in SMPTE Motion Imaging Journal, vol. 127, no. 6, pp. 1-7, July 2018. DOI: 10.5594/JMI.2018.2831078

[9] A. A. Haghighi, S. Shah Heydari and S. Shahbazpanahi, "Dynamic QoS-Aware Resource Assignment in Cloud-Based Content-Delivery Networks," in IEEE Access, vol. 6, pp. 2298-2309, 2018.

[10] G. Araniti, I. Bisio, M. De Sanctis, A. Orsino, and J. Cosmas, "Multimedia Content Delivery for Emerging 5G-Satellite Networks," in IEEE Transactions on Broadcasting, vol. 62, no. 1, pp. 10-23, March 2016. DOI: 10.1109/TBC.2015.2511625

[11] R. Bush, K. Patel, D. Ward, Extended Message Support for BGP, Internet Engineering Task Force (IETF), Request for Comments: 8654 ISSN: 2070-1721, October 2019.

[12] Borhani, M. Corpus Analysis Using Relaxed Conjugate Gradient Neural Network Training Algorithm. Neural Processing Letters, 50, 839-849, 2018.

[13] Borhani, M. Consecutive spatial-spectral framework for remote sensing image classification. Earth Sci Inform, 2019 https://doi.org/10.1007/s12145-019-00431-x

[14] Mostafa Borhani, Multi-label Log-Loss function using L-BFGS for document categorization, Engineering Applications of Artificial $\begin{array}{llll}\text { Intelligence, } & \text { Volume } & \text { 91, }\end{array}$ https://doi.org/10.1016/j.engappai.2020.103623

[15] Nima Ghasemloo, Mostafa Borhani, Soft Computing Modelling of Urban Evolution: Tehran Metropolis, International Journal of Interactive Multimedia and Artificial Intelligence, 2019, DOI: 10.9781/ijimai.2019.03

[16] M. Borhani, A Multicriteria Optimization for Flight Route Networks in Large-Scale Airlines Using Intelligent Spatial Information, International Journal of Interactive Multimedia \& Artificial Intelligence Mar2020, Vol. 6 Issue 1, p123-131. 9p, DOI: 10.9781/ijimai.2019.11.001 